\title{
Strain Hardening Behavior in Elongational Viscosity for Binary Blends of Linear Polymer and Crosslinked Polymer
}

\author{
Masayuki YAMAguchi ${ }^{\dagger}$ and Hiroshi MiYata \\ Yokkaichi Research Laboratory, TOSOH Corporation, \\ 1-8 Kasumi, Yokkaichi, Mie 510-8540, Japan
}

(Received August 24, 1999)

\begin{abstract}
Shear and elongational flow properties have been studied for the binary blends of a linear polymer, such as isotactic polypropylene (PP) and polystyrene (PS), and gel fraction of a crosslinked terpolymer composed of ethylene, 1-hexene, and ethylidene norbornene (gEHDM). It was found that the PP/gEHDM (97/3) blend exhibits marked strain hardening in the elongational viscosity, although the shear viscosity is almost the same as that of the pure PP. On the other hand, the PS/gEHDM (97/3) blend shows no strain hardening in the elongational viscosity.

KEY WORDS Rheological Property / Elongational Viscosity / Shear Viscosity / Polypropylene / Polystyrene / Polymer Blend / Constitutive Equation /
\end{abstract}

Rheological properties under elongational flow have been recognized to be very important for various processing, such as blow-molding, spinning, film processing, thermoforming, and foaming. ${ }^{1-5}$ Therefore, numerous studies on the elongational viscosity have been carried out for various polymer melts. In particular, much attention has been focused on the relationship between molecular characteristics and the strain hardening behavior in elongational viscosity. According to the previous studies, the polymers containing high molecular weight fraction exhibit strain hardening behavior in elongational viscosity, ${ }^{6-8}$ which is owing to the existence of the long time relaxation mechanism. Furthermore, comb-shaped and randomly branched polymers, which have long chain branching, also show marked strain hardening, ${ }^{9-13}$ which is ascribed to both long time relaxation mechanism and larger value of the damping function. ${ }^{14}$ Moreover, the elongational flow properties for polymer composites have been also investigated. Mewis and Metzner ${ }^{15}$ demonstrated that blending of long fibers into a polymer melt strongly enhances the elongational viscosity, although there is little increment of shear viscosity. Furthermore, they showed that their experimental results well agree with Batchelor's theory. ${ }^{16}$ The enhancement of the elongational viscosity has been also reported for the various polymer composites with glass fibers. ${ }^{17-19}$ It should be noted that the composites with long fiber, however, do not exhibit strain hardening behavior but show higher elongational viscosity at whole strain or elongational time region. Also for various kinds of polymer blends, the elongational flow properties have been studied extensively. According to them, it has been found that the immiscible polymer blends, in which there is long time relaxation mechanism due to deformation and/or diffusion of the dispersed phase, do not show marked strain hardening. ${ }^{8,20-22}$

In this paper, we discuss the rheological properties for the binary blends of a linear polymer and a crosslinked polymer and show that the blend of an isotactic polypropylene and a crosslinked terpolymer composed of ethylene, 1-hexene, and ethylidene norbornene, exhibits marked strain hardening in the elongational viscosity, although the amount of the crosslinked terpolymer is only $3 \mathrm{wt} \%$. Furthermore, the elongational viscosity of the blend is compared with the predicted ones using the constitutive equation proposed by Lodge. ${ }^{23}$

\section{EXPERIMENTAL}

\section{Materials and Blend Preparation}

Isotactic propylene homopolymer (PP) (Chisso Petrochemical Corp., CHISSO POLYPRO K1011) and polystyrene (PS) (Dainippon Ink and Chemicals, Inc., Dick Polystyrene CR2500) used in this study were commercial grade. The number and weight average molecular weights were $M_{n}=7.6 \times 10^{4}$ and $M_{w}=5.0 \times 10^{5}$ for the PP as polypropylene standard and $M_{n}=6.9 \times 10^{4}$ and $M_{w}=$ $1.8 \times 10^{5}$ for the PS as polystyrene standard. Ethylene-1hexene-ethylidene norbornene terpolymer (EHDM) used was produced by metallocene catalyst system. ${ }^{24}$ The composition of the EHDM determined from both ${ }^{13} \mathrm{C}$ NMR and ${ }^{1} \mathrm{H}$ NMR is as follows ; 30.2 mol\% of ethylene, $67.5 \mathrm{~mol} \%$ of 1 -hexene, and $2.3 \mathrm{~mol} \%$ of ethylidene norbornene. The molecular weights, which were determined from simultaneous measurements of gel permeation chromatography (GPC) and intrinsic viscosity using the universal calibration curve, ${ }^{25}$ were $M_{n}=1.3 \times 10^{5}$ and $M_{w}=1.8 \times 10^{5}$. The EHDM has no crystalline phase because 1-hexene content is much enough to hinder the crystallization of ethylene unit.

The EHDM was crosslinked by the following procedure; $0.3 \mathrm{wt} \%$ of $\alpha, \alpha^{\prime}$-Bis( $t$-butyl peroxy) di isopropyl benzene as a crosslinking agent was added into the EHDM in a $30 \mathrm{cc}$ static mixer (Toyoseiki, LaboPlastmill) at room temperature. Then the obtained compound was heated and crosslinked at $463 \mathrm{~K}$ for $15 \mathrm{~min}$ using a laboratory hot press. Furthermore, the crosslinked EHDM was immersed into $p$-xylene with BHT (2,6-di-t-butyl $p$-cresol) as a radical catcher for $24 \mathrm{~h}$ at room temperature in order to eliminate the sol fraction. The degree of swelling $q$ and the gel fraction $\phi_{\mathrm{g}}$ were calculated using the following equations ;

\footnotetext{
${ }^{\dagger}$ To whom correspondence should be addressed (Phone : +81-593-63-2652, Fax : +81-593-65-5205, e-mail : m_yama@tosoh.co.jp).
} 


$$
q=\frac{w_{\mathrm{s}}}{w_{\mathrm{g}}}, \quad \phi_{\mathrm{g}}=\frac{w_{\mathrm{g}}}{w_{\mathrm{w}}}
$$

where $w_{\mathrm{s}}, w_{\mathrm{g}}$, and $w_{\mathrm{w}}$ are the weights of swollen gel, dry gel, and sum of the dry gel and dry sol, respectively.

The network chain density $v_{\mathrm{e}}$ is estimated by the Flory-Rehner equation, ${ }^{26}$

$$
\mathcal{V}_{e}=-\frac{\ln \left(1-q^{-1}\right)+q^{-1}+\chi q^{-2}}{V_{1}\left(q^{-1 / 3}-0.5 q^{-1}\right)}
$$

where $V_{1}$ the partial molar volume of the swelling solvent and $\chi$ the Flory-Huggins interaction parameter.

The gel fraction of the crosslinked EHDM, we will abbreviate this to gEHDM here, was blended with the PP or the PS in the static mixer at $453 \mathrm{~K}$ for $10 \mathrm{~min}$ with 0.5 wt\% of BHT. The rotor rate was $30 \mathrm{rpm}$. The content of the gEHDM in the blends was $3 \mathrm{wt} \%$. We also prepared a blend of the PP or the PS with the uncrosslinked EHDM in an attempt to investigate the miscibility of the polymers. The content of the EHDM was $20 \mathrm{wt} \%$.

The obtained blends were melt pressed in a laboratory hot press at $463 \mathrm{~K}$ and at $10 \mathrm{MPa}$ for $15 \mathrm{~min}$, and then were quenched at $303 \mathrm{~K}$. The thickness of the compression-molded samples was adjusted to suitable thickness for measurements.

\section{Measurements}

Frequency dependence of oscillatory shear moduli in the molten state was measured using a cone-plate or a parallel-plate type rheometer (Rheometrics, Dynamic Stress Rheometer SR-2000). Measurements were carried out under a nitrogen atmosphere in order to avoid thermo-oxidative degradation. The parallel-plate type rheometer was used only for the measurements of the crosslinked polymer. The time-temperature superposition was applied to frequency dependence of oscillatory moduli at different temperatures in an attempt to determine the linear viscoelastic properties over a wide range of time scale.

Time variation of elongational viscosity $\eta_{\overrightarrow{\mathrm{E}}}^{-}(t, \dot{\varepsilon})$ was measured in a silicone oil bath using a Meissner-type rheometer (Toyoseiki, MELTEN Rheometer) at various strain rates $\dot{\varepsilon}$. Rod-like samples with diameter of $3-4$ $\mathrm{mm}$ and length of $180 \mathrm{~mm}$ were prepared by using a capillary rheometer (Toyoseiki, Capilograph) at $463 \mathrm{~K}$. The diameter of samples was recorded by a video camera during stretching.

Temperature dependence of oscillatory tensile moduli in the solid state was measured using a dynamic mechanical analyzer (Rheology, DVE-V4). The frequency used was $10 \mathrm{~Hz}$ and the heating rate was $2 \mathrm{~K} \mathrm{~min}^{-1}$. The rectangular specimens, in which the width is $3 \mathrm{~mm}$, the thickness is $0.5 \mathrm{~mm}$, and the length is $20 \mathrm{~mm}$, were used.

Morphology of the blends was studied by means of an AKASHI scanning electron microscope (SEM) model SIGMA-II and a JEOL transmission electron microscope (TEM) model JEM-2000 FX. Prior to the SEM observation, the surface of the cryogenically fractured samples was gold coated. For the TEM observation, the ultrathin films were sectioned into slices after being stained by ruthenium tetraoxide. (a)

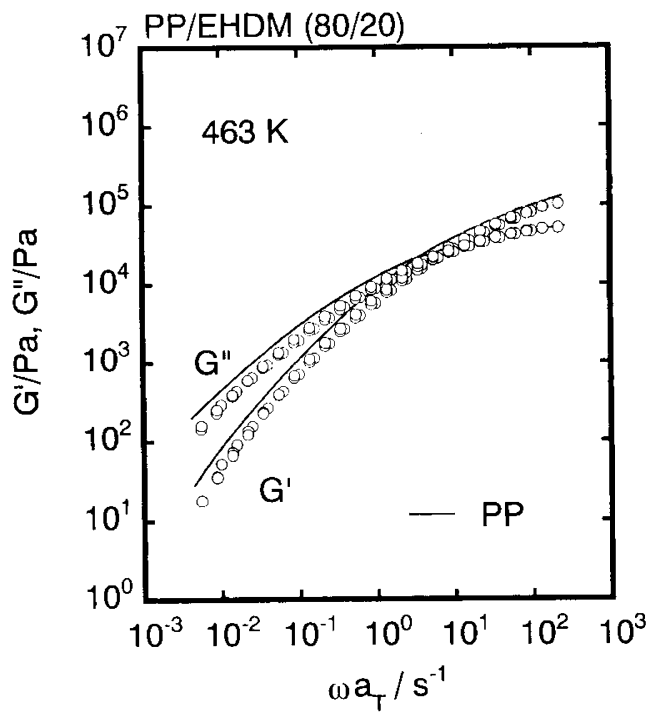

(b)

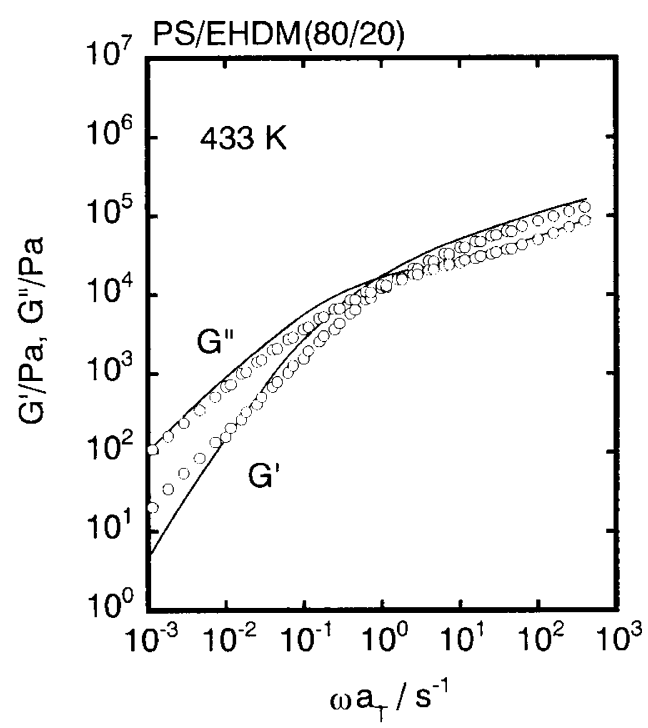

Figure 1. Master curves of frequency dependence of shear storage modulus $G^{\prime}$ and loss modulus $G^{\prime \prime}$ at $463 \mathrm{~K}$ for (a) PP/EHDM $(80 / 20)$ and (b) PS/EHDM (80/20). In the figure, solid lines represent those of (a) PP and (b) PS.

\section{RESULTS AND DISCUSSION}

\section{Miscibility with EHDM}

Prior to the investigation on the rheological properties for the blends with $\mathrm{gEHDM}$, the miscibility with the EHDM (uncrosslinked) was studied. It would be very important for the discussion on the elongational flow properties.

Figure 1a shows the master curves of frequency dependence of shear storage modulus $G^{\prime}$ and loss modulus $G$ " for the PP (solid lines) and the PP/EHDM (80/20) (open circles) at $463 \mathrm{~K}$. As seen in the figure, there is no evidence for the existence of the long time relaxation mechanism in the blend, which indicates entanglement slippage is the longest relaxation mechanism.

Figure 1b shows the master curves for the PS (solid lines) and the PS/EHDM (80/20) (open circles) at $433 \mathrm{~K}$. The blend shows the "secondary plateau" in the $G$ ' curve 
(a)
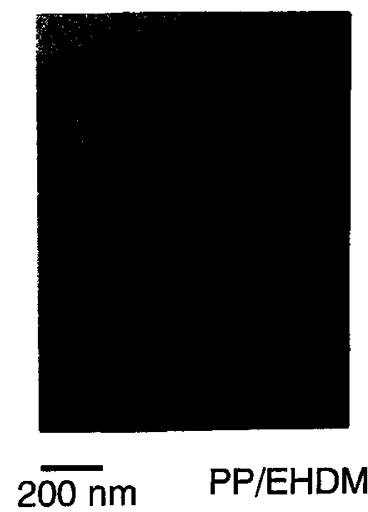

(b)

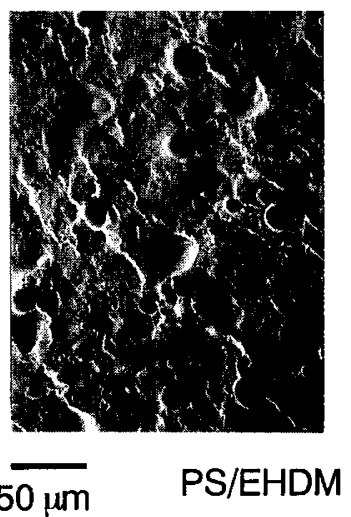

Figure 2. (a) TEM micrograph for PP/EHDM (80/20). (b) SEM micrograph for PS/EHDM (80/20).

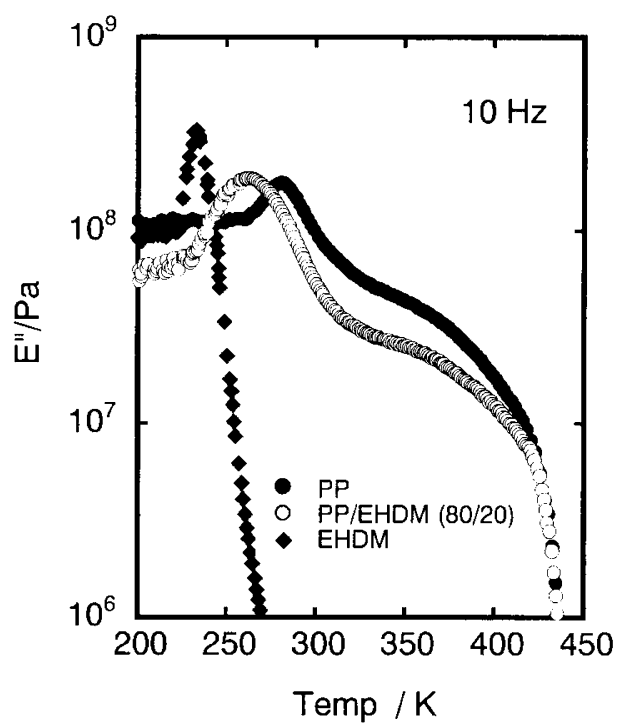

Figure 3. Temperature dependence of tensile loss modulus $E^{\prime \prime}$ for PP ( $)$, EHDM $(8)$, and PP/EHDM (80/20) (O). The frequency used was $10 \mathrm{~Hz}$.

at lower frequency region, which is ascribed to the long time relaxation mechanism related to the microheterogeneous structure. ${ }^{27-29}$

For the PP/EHDM (80/20), we cannot detect the phase separation by both TEM and SEM observation. Figure 2a exemplifies a TEM micrograph. On the other hand, two phase morphology is observed in the PS/EHDM (80/ 20 ) as shown in the SEM micrograph (Figure $2 b$ ). It is found from Figures $1 \mathrm{~b}$ and $2 \mathrm{~b}$ that the PS is immiscible with the EHDM, and the blend results in phase separation.

Figure 3 shows the temperature dependence of tensile loss modulus $E^{\prime \prime}$ for the PP, the EHDM, and the PP/ $\operatorname{EHDM}(80 / 20)$. There is only single peak in the $E^{\prime \prime}$ curve in the temperature region from 220 to $300 \mathrm{~K}$ in the blend. The peak temperature is between those of the pure components, i.e., glass transition temperatures of the PP and the EHDM. The result suggests that the EHDM used is miscible with the $\mathrm{PP}$ in the amorphous region.

According to our recent studies on the miscibility of

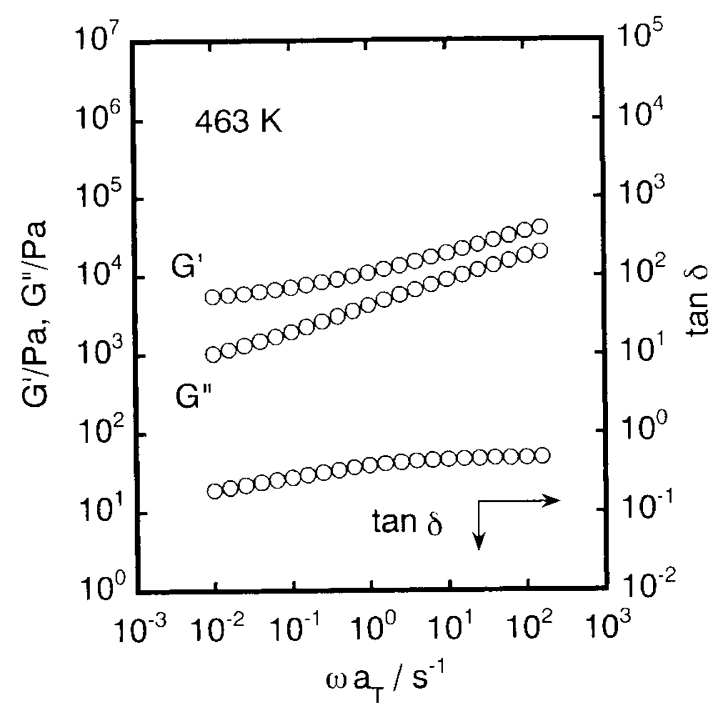

Figure 4. Frequency dependent of shear storage modulus $G^{\prime}$, loss modulus $G^{\prime \prime}$, and loss tangent $\tan \delta$ for gEHDM at $463 \mathrm{~K}$.

PP and ethylene- $\alpha$-olefin copolymers,,$^{29-31}$ the ethylene1 -hexene copolymer whose 1-hexene content is above 50 mol\% was found to be miscible with PP in the molten state. Figures 1-3 indicate that the incorporation of a small amount of ethylidene norbornene into the ethylene-1-hexene copolymer has no influence on the miscibility with PP.

\section{Characteristics of gEHDM}

The gel fraction of the crosslinked EHDM is found to be $76.3 \mathrm{wt} \%$ and the degree of swelling of the gel is 60.0 . Assuming that the Flory-Huggins interaction parameter $\chi$ between gEHDM and $p$-xylene is the same as that between $\mathrm{PP}$ and $p$-xylene, which is estimated from the solubility parameter $\delta_{i}$ of them by eq $3,32,33$ network chain density is estimated to be $4.52 \mathrm{~mol} \mathrm{~m}^{-3}$.

$$
\chi=\frac{v_{0}}{k_{\mathrm{B}} T}\left(\delta_{1}-\delta_{2}\right)^{2}
$$

where $v_{0}$ the reference volume and $k_{\mathrm{B}}$ the Boltzmann constant.

From the network chain density, the molecular weight between the crosslinking points is found to be $1.9 \times 10^{5}$. The value is much larger than the average molecular weight between the entanglement couplings by points. ${ }^{31}$

Recently, rheological measurements have been often carried out for the characterization of a gel. According to Chambon and Winter, ${ }^{34-36}$ scaling law governs the oscillatory moduli at the gel point,

$$
G^{\prime} \propto G^{\prime \prime} \propto \omega^{n} \quad(0<n<1)
$$

or

$$
\tan \delta=\tan (n \pi / 2)
$$

Beyond the gel point, $G^{\prime}$ at lower frequency region does not fully relax and equilibrium modulus is observed.

Figure 4 shows the frequency dependent of $G^{\prime}, G^{\prime \prime}$, and loss tangent $\tan \delta$ for the gEHDM. As seen in the 


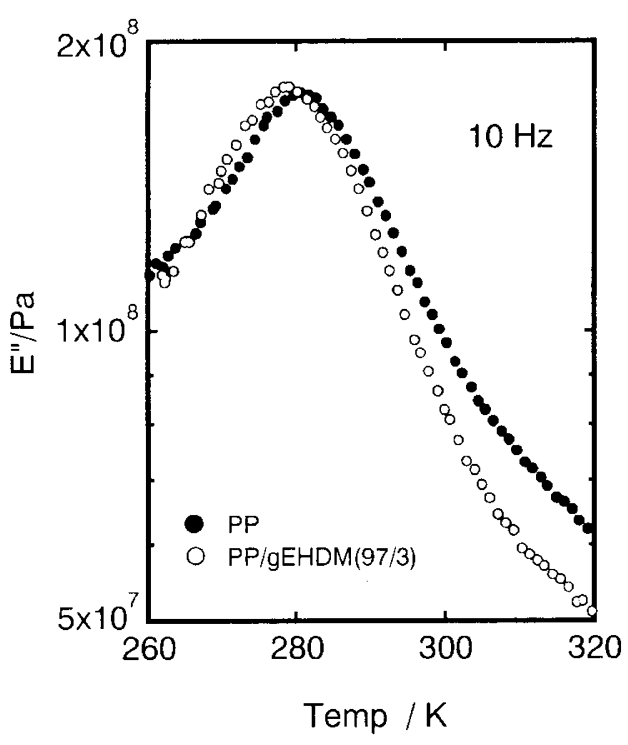

Figure 5. Temperature dependence of tensile loss modulus $E$ " for PP $(-)$ and PP/gEHDM (97/3) (O). The frequency used was 10 $\mathrm{Hz}$. (a)

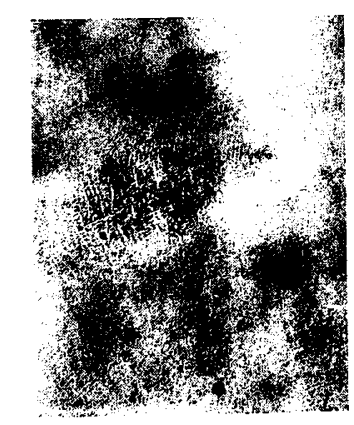

\section{$2 \overline{00 \mathrm{~nm}}$}

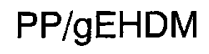

(b)

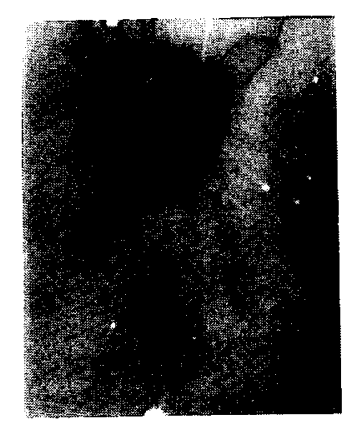

PS/gEHDM
Figure 6. (a) TEM micrograph for PP/gEHDM (97/3). (b) TEM micrograph for PS/gEHDM (97/3).

figure, $G^{\prime}$ is larger than $G^{\prime \prime}$, and the slope of $G^{\prime}$ at lower frequency region is small, although both $G^{\prime}$ and $G^{\prime \prime}$ decrease with decreasing the frequency. Therefore, it can be concluded that the gEHDM is just beyond the critical point of sol-gel transition from the rheological point of view.

\section{Morphology of the Blends with gEHDM}

Figure 5 shows the temperature dependence of $E^{\prime \prime}$ for the PP/gEHDM (97/3). The peak temperature of $E^{\prime \prime}$ for the PP/gEHDM is found to be lower than that for the PP. The result indicates the mutual dissolution of PP molecules and network chains of gEHDM.

Figure 6 shows TEM micrographs for the blends in which $3 \mathrm{wt} \%$ of gEHDM is contained. The samples were stained by ruthenium tetraoxide, therefore dark area represents the rubbery, i.e., gEHDM-rich, region. As seen in Figure 6a, there is a light-dark domain in the center of the micrograph, which represents the gEHDMrich region. Furthermore, it should be noted that there (a)

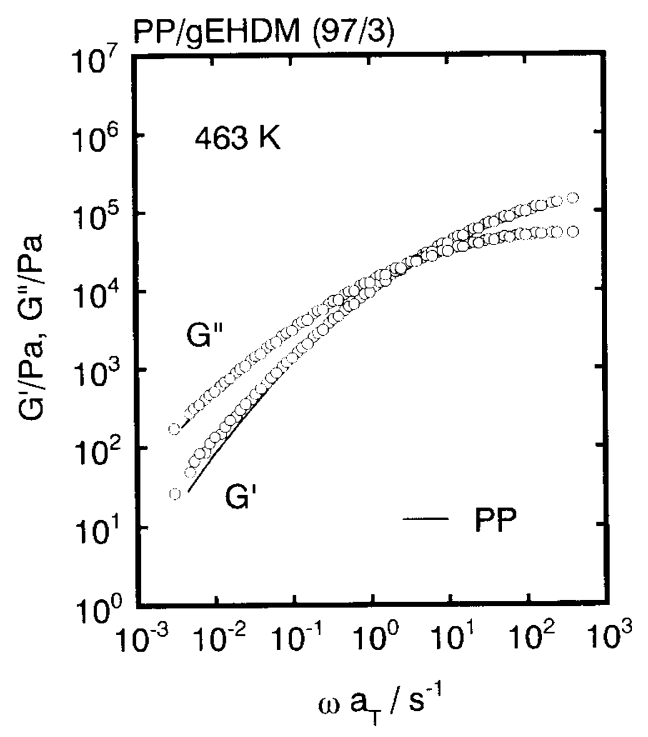

(b)

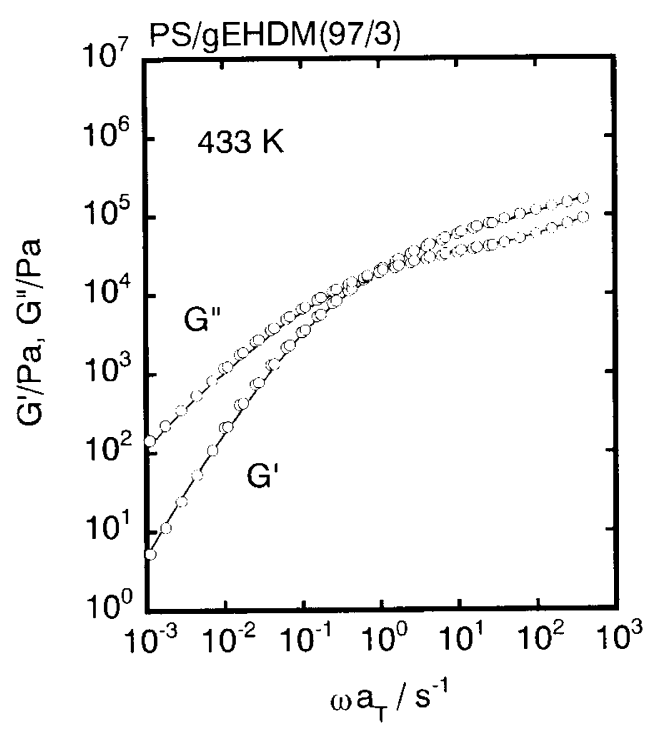

Figure 7. Master curves of frequency dependence of shear storage modulus $G^{\prime}$ and loss modulus $G^{\prime \prime}$ at $463 \mathrm{~K}$ for (a) PP/gEHDM (97/3) and (b) PS/gEHDM (97/3). In the figure, solid lines represent those of (a) PP and (b) PS.

are a lot of lamellae even in the dark region. These lamellae will be ascribed to the PP crystallites because gEHDM does not crystallize. The result demonstrates that PP molecules interpenetrate into the gEHDM during the blending. Consequently, gEHDM is swollen by the PP molecules, which corresponds with dynamic mechanical properties as shown in Figure 5 . The miscibility between the PP and the EHDM will be responsible for the swelling behavior.

On the other hand, gEHDM phase is dispersed in the PS matrix (Figure 6b) for the PS/gEHDM (97/3). The size of the dispersed phase is above 1 micron.

\section{Viscoelastic Properties under Shear Flow}

Figure 7a shows the master curves of frequency dependence of oscillatory shear moduli for the PP and the $\mathrm{PP} / \mathrm{gEHDM}(97 / 3)$ at $463 \mathrm{~K}$. It is confirmed prior to the measurements that the shear moduli are stable over $4 \mathrm{~h}$ at the experimental temperatures. Over the range of in- 
(a)

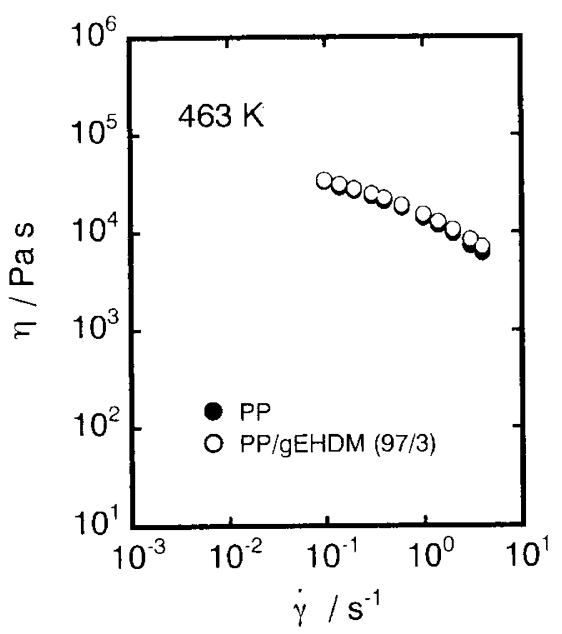

(b)

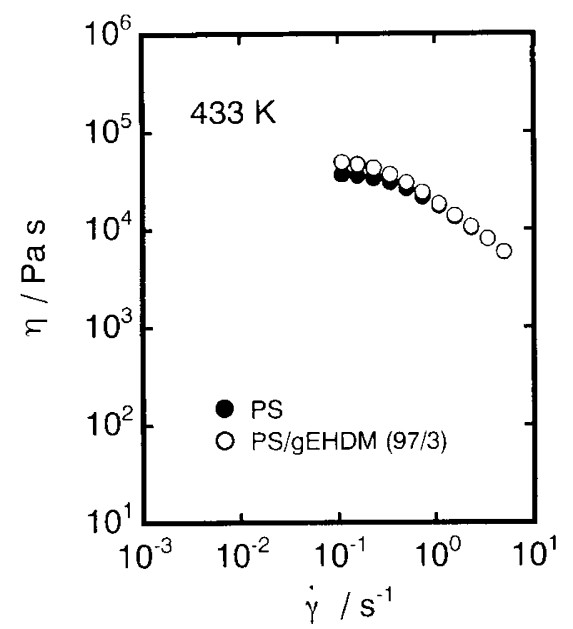

Figure 8. Shear rate dependence of shear stress measured by a cone-plate rheometer at $463 \mathrm{~K}$ for (a) PP (O) and PP/gEHDM (97/ 3) $(\bigcirc)$ and (b) PS $(\bigcirc)$ and PS/gEHDM (97/3) $(\bigcirc)$.

terest, the result was found to obey the principle of the time-temperature superposition. The apparent activation energy $\Delta H_{\mathrm{a}}$ of the blend $\left(40 \mathrm{~kJ} \mathrm{~mol}^{-1}\right)$, which is calculated from the temperature dependence of shift factor $a_{\mathrm{T}}$ using the Arrhenius equation, is the same as that of the pure PP. As seen in Figure $7 \mathrm{a}, G^{\prime}$ of the blend in the lower frequency region is larger than that of the pure PP. This is associated with the long time relaxation which will relate to the entanglement couplings between network chains of the miscible gEHDM and PP molecules. On the other hand, oscillatory moduli for the PS/gEHDM are the same as those for the pure PS as shown in Figure $7 \mathrm{~b}$. This is plausible because the amount of gEHDM, which behaves like a filler, is only $3 \mathrm{wt} \%$.

Shear rate dependence of steady-state shear viscosity $\eta(\dot{\gamma})$ is shown in Figure 8 . The curvature of the time variation of shear viscosity for the blends with gEHDM is almost the same as that for the pure PP or PS (but not presented here). It is found from Figure $8 \mathrm{a}$ that there is little difference in shear viscosity between the PP and the PP/gEHDM (97/3). The similar result is obtained for the PS/gEHDM (97/3) as shown in Figure $8 \mathrm{~b}$.

\section{Viscoelastic Properties under Elongational Flow}

Figure 9 shows the time variations of elongational vis- (a)

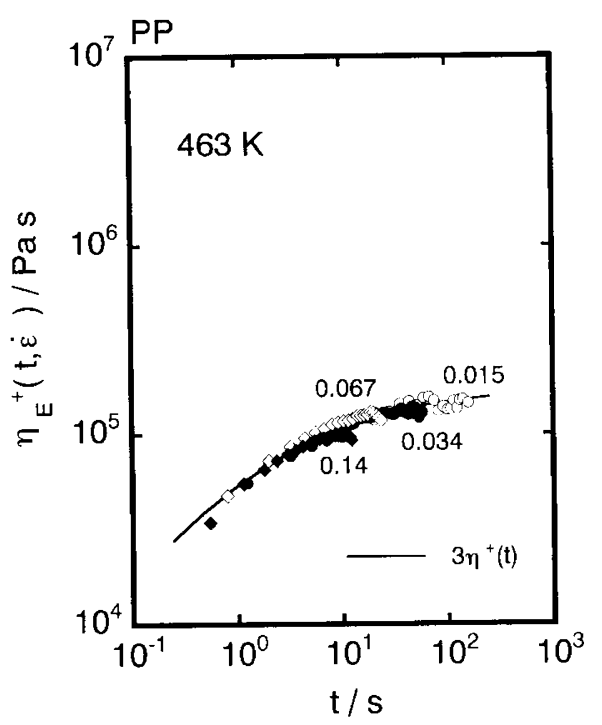

(b)

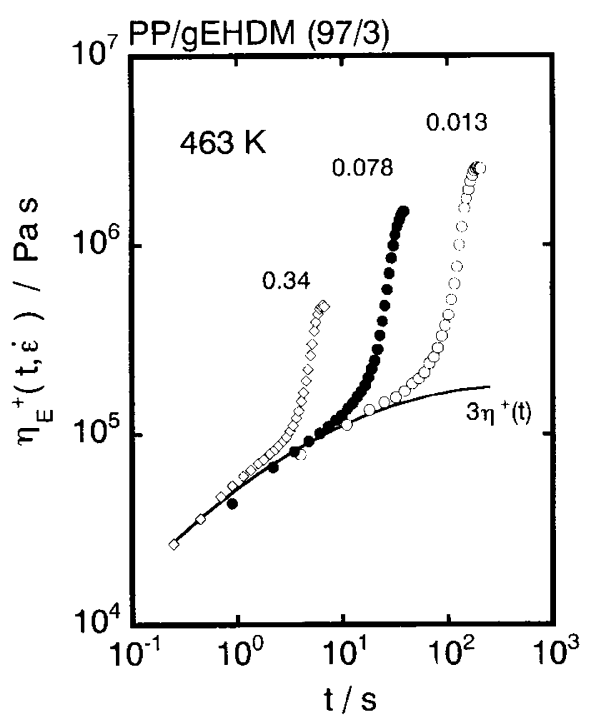

Figure 9. Time variations of elongational viscosity $\eta_{\mathrm{E}}^{+}(t, \dot{\varepsilon})$ at various elongational rate for (a) PP and (b) PP/gEHDM (97/3) at $463 \mathrm{~K}$. The numerals in the figure denote strain rate, and the solid line represents $3 r_{i}^{+}(t)$, where $r^{+}(t)$ is the time variation of the shear viscosity in the linear viscoelastic region.

cosity $\eta_{\mathrm{E}}^{+}(t, \dot{\varepsilon})$ at various elongational rate $\dot{\varepsilon}$ for the PP (Figure 9a) and the PP/gEHDM (97/3) (Figure 9b). It was confirmed of the uniform deformation from the time variation of the diameter of rod-like samples for both samples. The solid line in the figure represents $3 \eta^{+}(t)$, where $\gamma^{+}(t)$ is the time variation of the shear viscosity in the linear viscoelastic region calculated from the oscillatory shear moduli shown in Figure 6a, using an approximate equation proposed by Osaki et al., ${ }^{37}$

$$
\eta^{+}(t)=\left.t\left[G^{\prime \prime}(\omega)+1.12 G^{\prime}(\omega / 2)-0.200 G^{\prime}(\omega)\right]\right|_{\omega=1 / t}
$$

The elongational viscosity $\eta_{\mathrm{E}}^{+}(t, \dot{\varepsilon})$ for the PP/gEHDM much deviates from the curvature of $3 \eta^{+}(t)$ at high strain region. The maximum value in the elongational viscosity is about ten times as large as $3 \eta^{+}(t)$. On the other hand, the viscosity curves for the PP agree with $3 \eta^{+}(t)$ irrespective of the strain rates. No strain harden- 


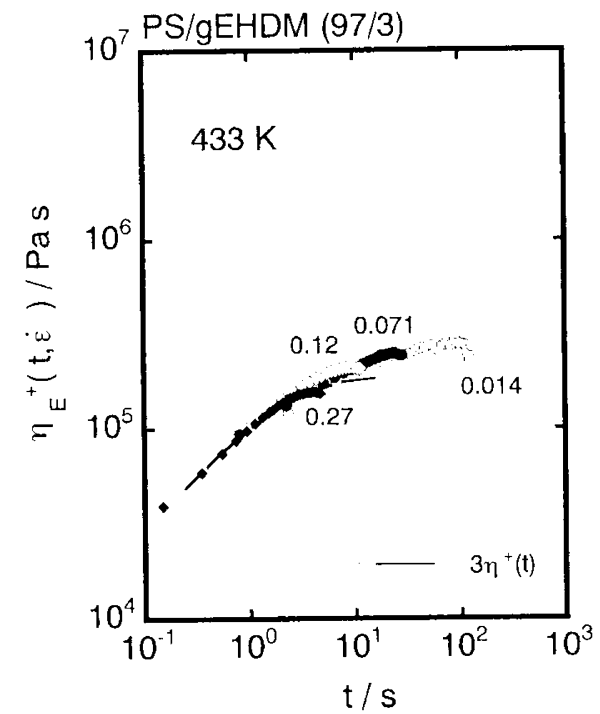

Figure 10. Time variations of elongational viscosity $\eta_{\mathrm{k}}^{\prime}(t, \dot{\varepsilon})$ at various elongational rate for PS/gEHDM (97/3) at $433 \mathrm{~K}$. The numerals in the figure denote strain rate, and the solid line represents $3 r_{i}^{-}(t)$, where $r_{t}^{+}(t)$ is the time variation of the shear viscosity in the linear viscoelastic region.

ing can be observed in the elongational viscosity, which is in good agreement with the previous study by Kurzbeck et al. ${ }^{38}$

Figure 10 shows $\gamma_{\mathrm{E}}^{+}(t, \dot{\varepsilon})$ for the PS/gEHDM (97/3). As seen in the figure, there is no strain hardening for the blend. Furthermore, the samples are easy to break. Large domains of gEHDM would hinder the uniform deformation of the samples.

\section{Comparison with Lodge Model}

According to Lodge model, ${ }^{23}$ the growth of uniaxial elongational viscosity is represented by the following equation,

$$
\begin{gathered}
\eta_{\mathrm{E}}^{+}(t, \dot{\varepsilon})=\sum_{i=1}^{N} A_{i}\left(3-2 \tau_{i} \mathcal{v} \exp (-u t)-\tau_{i} u \exp (-v t)\right) u^{-1} v^{-1} \\
u=\tau_{i}^{-1}-2 \dot{\varepsilon}, \quad v=\tau_{i}^{-1}+\dot{\varepsilon}
\end{gathered}
$$

where $A_{i}$ and $\tau_{i}$ are adjustable parameters to fit the oscillatory shear moduli. As well known, large values of elongational viscosity will be given by Lodge model, because the decrease in relaxation moduli in nonlinear region, i.e., decrease in damping function is not taken into account.

As seen in Figure 11, however, the degree of strain hardening in experimental elongational viscosity at a lower strain rate is larger than that calculated by Lodge model. The deviation from Lodge model will be attributed to the morphology in the blend. In particular, swelling behavior of gEHDM seems to play the decisive role on the elongational viscosity, because the trapped entanglements, i.e., entanglements between PP molecules and network chains, are responsible for the long time relaxation mechanism. As a result, strain hardening would take place. Therefore, the miscibility between a linear polymer and a crosslinked polymer strongly affects the

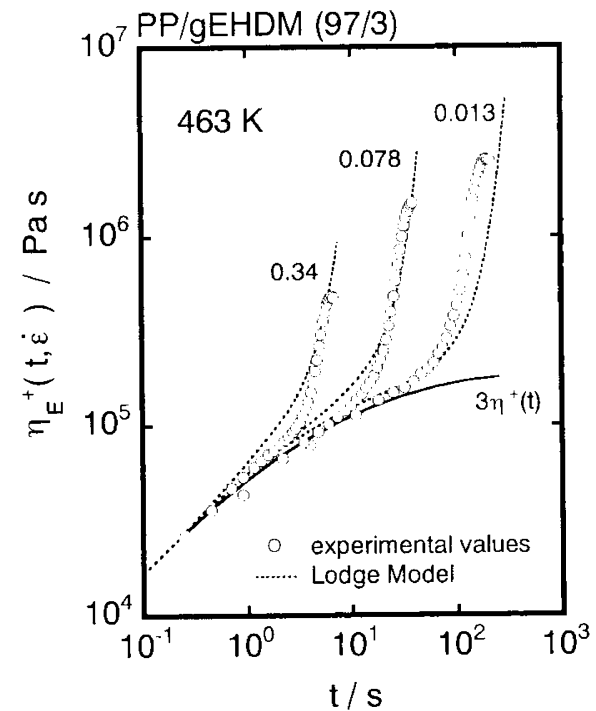

Figure 11. Time variations of elongational viscosity $r_{\mathrm{E}}^{+}(t, \dot{\varepsilon})$ at various elongational rate for PP/gEHDM (97/3) at $463 \mathrm{~K}$. The open symbols denote experimental values and dashed lines predicted values by Lodge model.

elongational viscosity. Moreover, present experimental results will give valuable information on the elongational viscosity of low density polyethylene (LDPE). As well known, Meissner ${ }^{39}$ and Mgill and Peddada ${ }^{40}$ suggested that in the IUPAC A LDPE, which exhibits marked strain hardening, ${ }^{9}$ ultrahigh molecular weight fraction and/or microgel are contained, which cannot be detected by GPC. The role of microgel on the rheological properties, however, remains to be unsolved. Further study on the present blend system will make it clear that the effect of the microgel on the elongational viscosity.

\section{CONCLUSIONS}

The rheological properties for the binary blends of a linear polymer, such as PP and PS, and gel fraction of a crosslinked EHDM (gEHDM) characterized as the gel which is just beyond the critical point of sol-gel transition. The PP/gEHDM (97/3) blend exhibits marked strain hardening in elongational viscosity, although the shear viscosity is almost the same as that of the pure PP. Moreover, it was found that the gEHDM in the PP/ gEHDM blend is swollen by the interpenetrating of the PP molecules. On the other hand, the PS/gEHDM (97/3) blend shows no strain hardening.

Acknowledgment. The authors are grateful to Professor Toshikazu Takigawa of Kyoto University, Professor Masaoki Takahashi of Kyoto Institute of Technology, and Professors Jan Mewis and Paula Moldenares of Katholieke Universiteit Leuven for their valuable suggestions and discussions. The authors also express their gratitude to Mr. Ryuji Ikeda of TOSOH for preparing the sample and Mr. Takayuki Adachi of TOSOH for microscope observation. 


\section{REFERENCES}

1. D. H. Sebastian, Polym. Eng. Sci., 23, 572 (1983).

2. H. Yamane and J. L. White, J. Soc. Rheol. Jpn., 15, 131 (1987).

3. M. Fleissner, Intern. Polym. Proc., 2, 229 (1988).

4. M. Shinohara, J. Soc. Rheol. Jpn., 19, 118 (1991).

5. T. Murata, J. Takimoto, and K. Koyama, J. Soc. Mat. Sci., Jpn., 45, 1300 (1996).

6. H. Münstedt, J. Rheol ., 24, 847 (1980).

7. H. Münstedt and H. M. Laun, Rheol. Acta, 20, 211 (1981).

8. T. Takahashi, J. Takimoto, and K. Koyama, J. Appl. Polym. Sci., 72, 961 (1999).

9. J. Meissner, Rheol. Acta, 10, 230 (1971).

10. K. Koyama and O. Ishizuka, Polym. Process Eng., 1, 55 (1983).

11. H. M. Laun and H. Schuch, J. Rheol., 33, 119 (1989).

12. K. Yoshikawa, N. Toneaki, Y. Moteki, M. Takahashi, and T. Masuda, J. Soc. Rheol. Jpn., 18, 80 (1990).

13. M. Sugimoto, T. Tanaka, Y. Masubuchi, J. Takimoto, and K. Koyama, J. Appl. Polym. Sci., 73, 1493 (1999).

14. K. Osaki, Rheol. Acta, 32, 429 (1993).

15. J. Mewis and A. B. Metzner, J. Fluid Mech., 62, 593 (1974).

16. G. K. Batchelor, J. Fluid Mech., 46, 813 (1971).

17. Y. Chan, Y. Oyanagi, and J. L. White, J. Rheol., 22, 507 (1978).

18. M. R. Kamal and L. A. Utracki, Polym. Comp., 5, 289 (1984).

19. H. M. Laun, Colloid Polym. Sci., 262, 257 (1984).

20. K. Min, J. L. White, and J. F. Fellers, J. Appl. Polym. Sci., 29, 2117 (1984).

21. L. A. Utracki and P. Sammut, Polym. Eng. Sci., 30, 1019 (1990).

22. T. Hattori, T. Takigawa, and T. Masuda, J. Soc. Rheol. Jpn.,
20, 141 (1992)

23. A. S. Lodge, "Elastic Liquids", Academic Press, London, 1964.

24. H. Miyata, M. Yamaguchi, and A. Akimoto, Proceeding of MetCon '96 Worldwide Metallocene Conference, Houston, June, 1996.

25. Z. Grubisic, P. Rempp, and H. Benoit, J. Polym. Sci., Polym. Lett. Ed ., 5, 753 (1967).

26. P. J. Flory, "Principles of Polymer Chemistry", Cornell University Press, Ithaca, New York, N.Y., 1953.

27. J. F. Palierne, Rheol. Acta, 29, 204 (1990).

28. D. Graebling, R. Muller, and J. F. Palierne, Macromolecules, 26, 320 (1993).

29. M. Yamaguchi and H. Miyata, Macromolecules, 32, 5911 (1999).

30. M. Yamaguchi, H. Miyata, K. Nitta, and T. Masuda, J. Appl. Polym. Sci., 63, 467 (1997).

31. M. Yamaguchi, K. Suzuki, and H. Miyata, J. Polym. Sci., Polym. Phys. Ed., 37, 701 (1999).

32. J. L. Gardon, Encycl. Polym. Sci. Technol ., 3, 833 (1965).

33. W. W. Graessley, R. Krishnamoorti, G. C. Reichart, N. P Balsara, L. J. Fetter, and D. J. Lohse, Macromolecules, 28, 1260 (1995)

34. F. Chambon and H. H. Winter, Polym. Bull., 13, 499 (1985).

35. H. H. Winter and F. Chambon, J. Rheol., 30, 367 (1986).

36. F. Chambon and H. H. Winter, J. Rheol ., 31, 683 (1987).

37. K. Osaki, A. Murai, N. Bessho, and B. S. Kim, J. Soc. Rheol. Jpn., 4, 166 (1976).

38. S. Kurzbeck, F. Oster, H. Münstedt, T. Q. Nguyen, and R. Gensler, J. Rheol., 43, 359 (1999).

39. J. Meissner, Pure Appl. Chem., 42, 553 (1975).

40. J. H. Magill and S. Peddada, J. Polym. Sci., Polym. Phys. Ed., 17, 1947 (1979) 